\title{
PERIODICITY OF THE PARITY OF A PARTITION FUNCTION RELATED TO MAKING CHANGE
}

\author{
PATRICK COSTELLO AND MICHAEL OSBORNE
}

\begin{abstract}
The solutions to a change problem form restricted partitions. For one particular change problem, we look at the sequence representing the parity of these restricted partition values. It appears that the period of this sequence has not been studied. Through recurrences involving binomial coefficients, we find that the sequence has a period of 200 .
\end{abstract}

\section{INTRODUCTION}

How many ways are there to give someone a certain amount of money $\mathrm{M}$ in change using pennies, nickels, dimes, and quarters? This would mean that $\mathrm{M}=$ $p * 1+n * 5+d * 10+q * 25$ where $p$ represents the number of pennies, $n$ represents the number of nickels, $d$ represents the number of dimes, and $q$ represents the number of quarters. In 1956, Polya [7] popularized a version of this problem where he included half-dollars. Since half-dollars seem to have gone away, we will look at the problem without half-dollars allowed.

The unrestricted partition function, usually denoted $p(n)$, represents the number of ways to write $n$ as a sum of positive integers. Let $p_{c}(n)$ represent the number of ways to make change for $n$ cents using pennies, nickels, dimes, and quarters. This is a restricted partition function where the only integers allowed in the sum are $1,5,10,25$. The question of determining the parity of the partition function was originally raised by S. Ramanujan [6, pp. 1087, 1098] but has not been settled (see [3]). Investigations into the parity of certain restricted partition functions have been undertaken by Blecksmith [1, Hirschhorn 4, and Lahiri [5]. These works have dealt with the situation where the summands are from specific residue classes of a given modulus. In this paper, we show that the parity of the sequence $\left\{p_{c}(n)\right\}$ is periodic, and that the period length is 200 .

\section{BASICS}

One quick observation about the function $p_{c}(n)$ is that each way to make change for $n=5 k$ gives precisely one way to make change for $5 k+1,5 k+2,5 k+3$, and $5 k+4$. You simply add on the appropriate number of pennies. Hence $p_{c}(5 k)=$ $p_{c}(5 k+1)=p_{c}(5 k+2)=p_{c}(5 k+3)=p_{c}(5 k+4)$.

Received by the editor August 2, 2007 and, in revised form, August 22, 2007.

2000 Mathematics Subject Classification. Primary 11P83.

Key words and phrases. Partitions, parity.

(C)2008 American Mathematical Society Reverts to public domain 28 years from publication 
If you have a function $f(n)$ defined on the nonnegative integers, we call the power series $\sum_{n=0}^{\infty} f(n) z^{n}$ the generating function of $f$. Looking at the generating function often turns out to be a useful way to find a closed or recursive formula for $f$. Graham, Knuth, and Patashnik 2 show the method for finding a solution to Polya's problem by writing the generating function as a product of known closed formulas for other power series, and doing some simplifying.

\section{Closed formula for values}

Following the method of Graham, Knuth, and Patashnik, we can find a formula for $p_{c}(n)$ by starting with the power series

$$
\begin{aligned}
& P=1+z+z^{2}+z^{3}+z^{4}+\cdots \quad \text { corresponding to pennies, } \\
& N=1+z^{5}+z^{10}+z^{15}+z^{20}+\cdots \quad \text { corresponding to nickels, } \\
& D=1+z^{10}+z^{20}+z^{30}+z^{40}+\cdots \quad \text { corresponding to dimes, } \\
& Q=1+z^{25}+z^{50}+z^{75}+z^{100}+\cdots \quad \text { corresponding to quarters. }
\end{aligned}
$$

Forming the product of these gives the generating function

$$
\sum_{n=0}^{\infty} p_{c}(n) z^{n}=P * N * D * Q=\frac{1}{1-z} \cdot \frac{1}{1-z^{5}} \cdot \frac{1}{1-z^{10}} \cdot \frac{1}{1-z^{25}} .
$$

Using that $\frac{1}{1-z}=\left(1+z+z^{2}+z^{3}+z^{4}\right) /\left(1-z^{5}\right)$, we can simplify the previous equation to

$$
\begin{aligned}
\sum_{n=0}^{\infty} p_{c}(n) z^{n} & =\frac{1+z+z^{2}+z^{3}+z^{4}}{1-z^{5}} \frac{1}{1-z^{5}} \frac{1}{1-z^{10}} \frac{1}{1-z^{25}} \\
& =\left(1+z+z^{2}+z^{3}+z^{4}\right) C\left(z^{5}\right)
\end{aligned}
$$

where

$$
C(z)=\frac{1}{1-z} \frac{1}{1-z} \frac{1}{1-z^{2}} \frac{1}{1-z^{5}} .
$$

Since each denominator is a divisor of $1-z^{10}$, we can write

$$
C(z)=\frac{A(z)}{\left(1-z^{10}\right)^{4}}
$$

where the polynomial $A(z)$ turns out to be exactly the same polynomial of degree 31 appearing on page 331 in Graham, et. al. [2]. Using that $\frac{1}{(1-z)^{4}}=\sum_{n=0}^{\infty}\left(\begin{array}{c}n+3 \\ 3\end{array}\right) z^{n}$, we have that

$$
\begin{aligned}
C(z) & =\frac{A(z)}{\left(1-z^{10}\right)^{4}} \\
& =A(z) \sum_{n=0}^{\infty}\left(\begin{array}{c}
n+3 \\
3
\end{array}\right) z^{10 n} .
\end{aligned}
$$

This means that the coefficient of $z^{10 q+r}$ with $0 \leq r<10$ in the power series $C(z)$ will be

$$
A_{r}\left(\begin{array}{c}
q+3 \\
3
\end{array}\right)+A_{10+r}\left(\begin{array}{c}
q+2 \\
3
\end{array}\right)+A_{20+r}\left(\begin{array}{c}
q+1 \\
3
\end{array}\right)+A_{30+r}\left(\begin{array}{l}
q \\
3
\end{array}\right)
$$


Finally,

$$
p_{c}(50 q+5 r+s)=A_{r}\left(\begin{array}{c}
q+3 \\
3
\end{array}\right)+A_{10+r}\left(\begin{array}{c}
q+2 \\
3
\end{array}\right)+A_{20+r}\left(\begin{array}{c}
q+1 \\
3
\end{array}\right)+A_{30+r}\left(\begin{array}{l}
q \\
3
\end{array}\right)
$$

for any nonnegative $q$ and $0 \leq r<10$ and $0 \leq s<5$. For example, $\mathrm{M}=1039=$ $50 * 20+5 * 7+4$ and so

$$
\begin{aligned}
p_{c}(1039) & =A_{7}\left(\begin{array}{c}
23 \\
3
\end{array}\right)+A_{17}\left(\begin{array}{c}
22 \\
3
\end{array}\right)+A_{27}\left(\begin{array}{c}
21 \\
3
\end{array}\right)+A_{37}\left(\begin{array}{c}
20 \\
3
\end{array}\right) \\
& =24 * 1771+67 * 1540+9 * 1330 \\
& =157654 .
\end{aligned}
$$

This agrees with the coefficient of $z^{1039}$ when Mathematica expands the product

$$
\left(\sum_{n=0}^{1040} z^{n}\right)\left(\sum_{n=0}^{1040 / 5} z^{5 n}\right)\left(\sum_{n=0}^{1040 / 10} z^{10 n}\right)\left(\sum_{n=0}^{1040 / 25} z^{25 n}\right) .
$$

\section{ReCURREnCE FORMula FOR VALUeS}

Going back to equation (11), we can clear denominators and we get

$$
\left(\sum_{n=0}^{\infty} p_{c}(n) z^{n}\right)(1-z)\left(1-z^{5}\right)\left(1-z^{10}\right)\left(1-z^{25}\right)=1 .
$$

Expanding the product $(1-z)\left(1-z^{5}\right)\left(1-z^{10}\right)\left(1-z^{25}\right)$ gives

$$
\begin{aligned}
\left(\sum_{n=0}^{\infty} p_{c}(n) z^{n}\right)\left(1-z-z^{5}+z^{6}-z^{10}+z^{11}+z^{15}-z^{16}-z^{25}+z^{26}\right. & \\
& \left.+z^{30}-z^{31}+z^{35}-z^{36}-z^{40}+z^{41}\right)=1 .
\end{aligned}
$$

\begin{tabular}{|c|c|c|c|c|c|c|c|c|c|c|c|c|c|c|c|c|c|}
\hline$n$ & 0 & 1 & 2 & & 3 & 4 & 5 & & 6 & 7 & 8 & 9 & & 0 & 11 & 12 & 13 \\
\hline$p_{c}(n)$ & 1 & 1 & 1 & & 1 & 1 & 2 & & 2 & 2 & 2 & 2 & 4 & 4 & 4 & 4 & 4 \\
\hline$n$ & & 14 & 15 & 16 & 1 & 7 & 18 & 19 & 20 & & 21 & 22 & 23 & 24 & 2 & & 26 \\
\hline$p_{c}(n$ & & 4 & 6 & 6 & 6 & & 6 & 6 & 9 & 9 & 9 & 9 & 9 & 9 & 1 & 3 & 13 \\
\hline
\end{tabular}

For any $n>40$, we can equate the coefficients of $z^{n}$,

$$
\begin{aligned}
p_{c}(n) & -p_{c}(n-1)-p_{c}(n-5)+p_{c}(n-6)-p_{c}(n-10)+p_{c}(n-11) \\
& +p_{c}(n-15)-p_{c}(n-16)-p_{c}(n-25)+p_{c}(n-26)+p_{c}(n-30) \\
& -p_{c}(n-31)+p_{c}(n-35)-p_{c}(n-36)-p_{c}(n-40)+p_{c}(n-41)=0,
\end{aligned}
$$

which gives the recurrence

$$
\begin{aligned}
& p_{c}(n)=p_{c}(n-1)+p_{c}(n-5)-p_{c}(n-6)+p_{c}(n-10)-p_{c}(n-11) \\
& -p_{c}(n-15)+p_{c}(n-16)+p_{c}(n-25)-p_{c}(n-26)-p_{c}(n-30) \\
& \quad+p_{c}(n-31)-p_{c}(n-35)+p_{c}(n-36)+p_{c}(n-40)-p_{c}(n-41)
\end{aligned}
$$

with initial values

\begin{tabular}{|l|l|l|l|l|l|l|l|l|l|l|l|l|l|l|}
\hline$n$ & 27 & 28 & 29 & 30 & 31 & 32 & 33 & 34 & 35 & 36 & 37 & 38 & 39 & 40 \\
\hline$p_{c}(n)$ & 13 & 13 & 13 & 18 & 18 & 18 & 18 & 18 & 24 & 24 & 24 & 24 & 24 & 31 \\
\hline
\end{tabular}




\section{Simpler ReCURREnce}

In order to determine the period of the sequence $\left\{p_{c}(n)(\bmod 2)\right\}$, we could use this recurrence and sequentially compute lots of values mod 2 and hope to observe the pattern. A more effective approach takes advantage of the fact that $p_{c}(5 k)=$ $p_{c}(5 k+1)=p_{c}(5 k+2)=p_{c}(5 k+3)=p_{c}(5 k+4)$.

We want to look at just $5 k$ as input to the function. Then the recurrence says

$$
\begin{aligned}
& p_{c}(5 k)=p_{c}(5 k-1)+p_{c}(5 k-5)-p_{c}(5 k-6)+p_{c}(5 k-10)-p_{c}(5 k-11) \\
& -p_{c}(5 k-15)+p_{c}(5 k-16)+p_{c}(5 k-25)-p_{c}(5 k-26)-p_{c}(5 k-30) \\
& \quad+p_{c}(5 k-31)-p_{c}(5 k-35)+p_{c}(5 k-36)+p_{c}(5 k-40)-p_{c}(5 k-41) .
\end{aligned}
$$

Using the fact that for any $k$ the 5 consecutive values $p_{c}(5 k), p_{c}(5 k+1), p_{c}(5 k+2)$, $p_{c}(5 k+3), p_{c}(5 k+4)$ are identical, we can simplify the above recurrence to the much shorter one below:

$$
\begin{aligned}
p_{c}(5 k)=2 p_{c}(5 k-5)-2 p_{c}(5 k-15) & +p_{c}(5 k-16)+p_{c}(5 k-25) \\
& -2 p_{c}(5 k-30)+2 p_{c}(5 k-40)-p_{c}(5 k-41) .
\end{aligned}
$$

We can also get all inputs to be multiples of 5 using the fact that

$$
p_{c}(5 k-16)=p_{c}(5 k-20) \quad \text { and } \quad p_{c}(5 k-41)=p_{c}(5 k-45) .
$$

We now have a recurrence for inputs which are multiples of five involving just previous multiples of five:

$$
\begin{aligned}
p_{c}(5 k)=2 p_{c}(5 k-5)-2 p_{c}(5 k-15) & +p_{c}(5 k-20)+p_{c}(5 k-25) \\
& -2 p_{c}(5 k-30)+2 p_{c}(5 k-40)-p_{c}(5 k-45)
\end{aligned}
$$

or

$$
\begin{aligned}
p_{c}(5 k)=2 p_{c}(5(k-1))-2 p_{c}( & (k-3))+p_{c}(5(k-4))+p_{c}(5(k-5)) \\
& -2 p_{c}(5(k-6))+2 p_{c}(5(k-8))-p_{c}(5(k-9)) .
\end{aligned}
$$

If we sequentially compute a table of values for inputs which are consecutive multiples of five, we can use this formula to find the value of the function at any input. For example,

$$
\begin{aligned}
p_{c}(48) & =p_{c}(5 * 9+3)=p_{c}(5 * 9) \\
& =2 p_{c}(5(8))-2 p_{c}(5(6))+p_{c}(5(5))+p_{c}(5(4))-2 p_{c}(5(3))+2 p_{c}(5(1))-p_{c}(5(0)) \\
& =2 * 31-2 * 18+13+9-2 * 6+2 * 2-1 \\
& =39 .
\end{aligned}
$$

\section{RECURRENCE MOD 2}

Since many of the coefficients in the recurrence for inputs which are multiples of five happen to be two, we get a very short recurrence when we consider it mod 2 . The recurrence becomes

$$
p_{c}(5 k) \equiv p_{c}(5(k-4))+p_{c}(5(k-5))+p_{c}(5(k-9)) \quad(\bmod 2)
$$

with initial values mod 2 being

\begin{tabular}{|l||l|l|l|l|l|l|l|l|l|}
\hline$k$ & 0 & 1 & 2 & 3 & 4 & 5 & 6 & 7 & 8 \\
\hline \hline$p_{c}(5 k)(\bmod 2)$ & 1 & 0 & 0 & 0 & 1 & 1 & 0 & 0 & 1 \\
\hline
\end{tabular}


Using these and sequentially computing values, we observe a repeating pattern of

$$
\begin{aligned}
& 1,0,0,0,1,1,0,0,1,1,1,0,1,1,1,1,1,1,1,1, \\
& 0,1,1,1,0,0,1,1,0,0,0,1,0,0,0,0,0,0,0,0 .
\end{aligned}
$$

So the period in the recurrence for multiples of five seems to be 40 which would give a period of 200 in the original sequence $\left\{p_{c}(n)(\bmod 2)\right\}$. What is interesting to note from the repeating sequence is that the second twenty values are just the first twenty with one added mod 2 . This will be verified in the next section.

\section{Conclusion}

Define a new function $p_{s}(n)$ recursively by

$$
p_{s}(n) \equiv p_{s}(n-4)+p_{s}(n-5)+p_{s}(n-9) \quad(\bmod 2)
$$

with the initial values as given in the last table.

Let $f(z)=\sum_{n=0}^{\infty} p_{s}(n) z^{n}$. Then the recurrence says that

$$
\begin{aligned}
\sum_{n=9}^{\infty} p_{s}(n) z^{n} \equiv & z^{4} \sum_{n=9}^{\infty} p_{s}(n-4) z^{n-4}+z^{5} \sum_{n=9}^{\infty} p_{s}(n-5) z^{n-5} \\
& +z^{9} \sum_{n=9}^{\infty} p_{s}(n-9) z^{n-9} \quad(\bmod 2) .
\end{aligned}
$$

Using the initial values of $p_{s}(n)$, we have

$f(z)-\left(1+z^{4}+z^{5}+z^{8}\right) \equiv z^{4}\left(f(z)-\left(1+z^{4}\right)\right)+z^{5}(f(z)-(1))+z^{9} f(z) \quad(\bmod 2)$ which gives

$$
f(z)\left(1+z^{4}+z^{5}+z^{9}\right) \equiv\left(1+z^{4}+z^{5}+z^{8}\right)-z^{4}\left(1+z^{4}\right)-z^{5}(1) \equiv 1 \quad(\bmod 2) .
$$

Factoring the polynomial $\left(1+z^{4}+z^{5}+z^{9}\right)$ in $\mathbf{Z}_{2}[z]$, we have

$$
\left(1+z^{4}+z^{5}+z^{9}\right) \equiv(1+z)^{5}\left(1+z+z^{2}+z^{3}+z^{4}\right) \quad(\bmod 2) .
$$

Then

$$
f(z) \equiv \frac{1}{(1+z)^{5}} \frac{1}{1+z+z^{2}+z^{3}+z^{4}} \equiv \frac{1}{(1+z)^{5}} \frac{1-z}{1-z^{5}} \quad(\bmod 2) .
$$

Now we make use of the generating function identities

$$
\frac{1}{(1-z)^{5}}=\sum_{n=0}^{\infty}\left(\begin{array}{c}
n+4 \\
4
\end{array}\right) z^{n} \quad \text { and } \quad \frac{1}{1-z^{5}}=\sum_{n=0}^{\infty} z^{5 n}
$$

and arrive at

$$
f(z) \equiv(1+z)\left(\sum_{n=0}^{\infty}\left(\begin{array}{c}
n+4 \\
4
\end{array}\right) z^{n}\right)\left(\sum_{n=0}^{\infty} z^{5 n}\right) \quad(\bmod 2) .
$$

In the product $(1+z)\left(\sum_{n=0}^{\infty}\left(\begin{array}{c}n+4 \\ 4\end{array}\right) z^{n}\right)$ the coefficient of $z^{n}$ would be $\left(\begin{array}{c}n+4 \\ 4\end{array}\right)+\left(\begin{array}{c}n+3 \\ 4\end{array}\right)$. When we multiply by the series $\sum_{n=0}^{\infty} z^{5 n}$, the coefficient of $z^{n}$ would be

$$
\begin{aligned}
\left(\begin{array}{c}
n+4 \\
4
\end{array}\right) & +\left(\begin{array}{c}
n+3 \\
4
\end{array}\right)+\left(\begin{array}{c}
n+4-5 \\
4
\end{array}\right)+\left(\begin{array}{c}
n+3-5 \\
4
\end{array}\right) \\
& +\left(\begin{array}{c}
n+4-10 \\
4
\end{array}\right)+\left(\begin{array}{c}
n+3-10 \\
4
\end{array}\right)+\cdots
\end{aligned}
$$


Since the coefficient of $z^{n}$ in $f(z)$ is the function $p_{s}(n)$, we get

$$
p_{s}(n) \equiv\left(\begin{array}{c}
n+4 \\
4
\end{array}\right)+\left(\begin{array}{c}
n+3 \\
4
\end{array}\right)+p_{s}(n-5) \quad(\bmod 2) .
$$

Now let $b(n)=\left(\begin{array}{c}n+4 \\ 4\end{array}\right)+\left(\begin{array}{c}n+3 \\ 4\end{array}\right)$. By induction, it can be shown that

$$
b(n) \equiv\left\{\begin{array}{lll}
0 & (\bmod 2) & \text { if } n \equiv 1,2,3 \quad(\bmod 4) \\
1 & (\bmod 2) & \text { if } n \equiv 0 \quad(\bmod 4)
\end{array}\right.
$$

Substituting $b(n)$ into the recurrence (2) and applying it 4 times, we get

$$
\begin{aligned}
p_{s}(n) & \equiv p_{s}(n-5)+b(n) \\
& \equiv p_{s}(n-10)+b(n-5)+b(n) \\
& \equiv p_{s}(n-15)+b(n-10)+b(n-5)+b(n) \\
& \equiv p_{s}(n-20)+b(n-15)+b(n-10)+b(n-5)+b(n) .
\end{aligned}
$$

Since 5 and 4 are relatively prime, exactly one of the four values $n-15, n-10$, $n-5, n$ is divisible by 4 . Hence (3) shows that

$$
p_{s}(n) \equiv p_{s}(n-20)+1 \quad(\bmod 2) .
$$

This shows what we observed before, that the next twenty values are just the previous twenty values with one added mod 2. Applying this congruence twice we get

$$
p_{s}(n) \equiv p_{s}(n-40)+1+1 \equiv p_{s}(n-40) \quad(\bmod 2),
$$

which means this sequence has a period of 40 when considered mod 2.

So the period in the sequence $\left\{p_{c}(5 k)(\bmod 2)\right\}$ has a period of 40 which would imply a period of 200 in the original sequence $\left\{p_{c}(n)(\bmod 2)\right\}$. That is to say, we have proved the following theorem: the parity of the function $p_{c}(n)$ is periodic, and the length of the period is 200 .

\section{REFERENCES}

1. R. Blecksmith, J. Brillhart, and I. Gerst, Parity results for certain partition functions and identities similar to theta functions, Mathematics of Computation, 48 (177) (1987), pp. 29-38. MR866096 (87k:11113)

2. R. Graham, D. Knuth, and O. Patashnik, Concrete Mathematics, Addison-Wesley, Reading, MA, 1990. MR1397498 (97d:68003)

3. M. D. Hirschhorn, On the residue $\bmod 2$ and $\bmod 4$ of $p(n)$, Acta Arith., 38 (1980), pp. 105-109. MR604226 (82d:10025)

4. M. D. Hirschhorn, Parity results for certain partition functions, Ramanujan Journal, 4 (2000), pp. 129-135. MR1782196 (2001i:11121)

5. D. B. Lahiri, Some restricted partition functions: Congruences modulo 2, Trans. of the American Mathematical Society, 147 (1970), pp. 271-278. MR0260694(41:5318)

6. P. A. MacMahon, Collected Papers, vol. 1, MIT Press, Cambridge, MA, 1978. MR514405 (80k:01065)

7. G. Polya, On picture-writing, American Mathematical Monthly, 63 (1956), pp. 689-697. MR0081865(18:458b)

Department of Mathematics and Statistics, Eastern Kentucky University, Richmond, KENTUCKY 40475-3102

E-mail address: pat.costello@eku.edu

Department of Mathematics and Statistics, Eastern Kentucky University, Richmond, KENTUCKY 40475-3102 